\title{
A NEW FAMILY OF DAI-LIAO CONJUGATE GRADIENT METHODS WITH MODIFIED SECANT EQUATION FOR UNCONSTRAINED OPTIMIZATION
}

\author{
YutAO ZHENG*(D)
}

\begin{abstract}
In this paper, a new family of Dai-Liao-type conjugate gradient methods are proposed for unconstrained optimization problem. In the new methods, the modified secant equation used in [H. Yabe and M. Takano, Comput. Optim. Appl. 28 (2004) 203-225] is considered in Dai and Liao's conjugacy condition. Under some certain assumptions, we show that our methods are globally convergent for general functions with strong Wolfe line search. Numerical results illustrate that our proposed methods can outperform some existing ones.
\end{abstract}

Mathematics Subject Classification. 65K05, 90C26, 90C30.

Received May 19, 2021. Accepted October 16, 2021.

\section{INTRODUCTION}

Consider the following unconstrained optimization problem

$$
\min f(x), x \in \mathbb{R}^{n},
$$

where the objective function $f: \mathbb{R}^{n} \rightarrow \mathbb{R}$ is continuously differentiable and its gradient $g(x)$ is available. The problem (1.1) has a wide range of applications in areas of scientific computing and engineering. Therefore, its efficient and effective numerical solution methods have been intensively studied in the literature, including the spectral gradient methods $[5,15]$, conjugate gradient methods $[4,13]$ and memoryless BFGS methods [16]. Among them, conjugate gradient methods are popular and efficient for solving (1.1), especially for large scale problems.

Let $x_{k}$ be the $k$ th iterate point, $g_{k}$ the gradient of $f(x)$ at $x_{k}$, i.e. $g_{k}=g\left(x_{k}\right)$. The (nonlinear) conjugate gradient method is given by

$$
x_{k+1}=x_{k}+\alpha_{k} d_{k},
$$

where $\alpha_{k}$ is the step length computed by carrying out an one-dimension line search and $d_{k}$ is the search direction defined by

$$
d_{k}= \begin{cases}-g_{k}, & \text { if } k=0 \\ -g_{k}+\beta_{k} d_{k-1}, & \text { if } k \geq 1\end{cases}
$$

Keywords. Conjugate gradient method, Dai-Liao-type method, modified secant equation.

School of Mathematics and Information Science, Henan Normal University, Xinxiang 453007, PR China.

* Corresponding author: zhengyutao@htu.edu.cn

(C) The authors. Published by EDP Sciences, ROADEF, SMAI 2021 
where $\beta_{k}$ is a scalar.

Since exact line search for searching $\alpha_{k}$ is usually expensive and impractical, the strong Wolfe inexact line search is often considered in the convergence analysis and implementation of nonlinear conjugate gradient methods. It aims to find a step size $\alpha_{k}$ satisfying the following two strong Wolfe conditions

$$
\begin{aligned}
f\left(x_{k}+\alpha_{k} d_{k}\right) & \leq f\left(x_{k}\right)+\rho g_{k}^{T} d_{k}, \\
\left|g_{k+1}^{T} d_{k}\right| & \leq \sigma\left|g_{k}^{T} d_{k}\right|,
\end{aligned}
$$

where $0<\rho<\sigma<1$.

Nonlinear conjugate gradient method for unconstrained optimization problem is generated from the linear conjugate gradient method for a special quadratic minimization problem

$$
\min \frac{1}{2} x^{T} Q x+b^{T} x+c
$$

or its equivalent line system $Q x=b$, where $Q$ is a real symmetric positive definite matrix. Linear conjugate gradient methods generate a search direction such that the conjugacy condition holds, namely,

$$
d_{i}^{T} Q d_{j}=0, \forall i \neq j .
$$

For general nonlinear functions, it follows from the mean value theorem that there exists some $\tau \in(0,1)$ such that

$$
d_{k}^{T} y_{k-1}=\alpha_{k-1} d_{k}^{T} \nabla^{2} f\left(x_{k-1}+\tau \alpha_{k-1} d_{k-1}\right) d_{k-1},
$$

where $y_{k-1}=g_{k}-g_{k-1}$ denotes the gradient change. Therefore, it is reasonable to replace (1.6) by the following conjugacy condition:

$$
d_{k}^{T} y_{k-1}=0
$$

Let $d_{k}=-g_{k}+\beta_{k} d_{k-1}$ satisfy the above condition, we have the famous Hestenes-Stiefel formula [14]

$$
\beta_{k}^{H S}=\frac{g_{k}^{T} y_{k-1}}{y_{k-1}^{T} d_{k-1}} .
$$

In 2001, Dai and Liao [6] suggested an extended one

$$
d_{k}^{T} y_{k-1}=\operatorname{tg}_{k}^{T} s_{k-1},
$$

which leads to the following conjugate gradient parameter

$$
\beta_{k}^{\mathrm{DL}+}=\max \left\{\frac{g_{k}^{T} y_{k-1}}{y_{k-1}^{T} d_{k-1}}, 0\right\}-t \frac{g_{k}^{T} s_{k-1}}{d_{k-1}^{T} y_{k-1}},
$$

where $t>0$ is a scalar, $s_{k-1}=x_{k}-x_{k-1}$. Note that the first item has been restricted to be nonnegative like [10]. The DL+ method (1.2)-(1.3) with $\beta_{k}$ in (1.9) is globally convergent for general functions under the sufficient descent condition

$$
g_{k}^{T} d_{k} \leq-c\left\|g_{k}\right\|^{2}, c>0
$$

and some other suitable conditions, where and hereafter $\|\cdot\|$ denotes the Euclidean norm of vectors.

As a special case of Dai-Liao-type conjugate gradient method, the efficient CG_descent method [12] utilizes a particular $t$. The conjugacy parameter of CG_descent method is

$$
\beta_{k}^{N}=\frac{g_{k}^{T} y_{k-1}}{y_{k-1}^{T} d_{k-1}}-2 \frac{\left\|y_{k-1}\right\|^{2}}{s_{k-1}^{T} y_{k-1}} \cdot \frac{g_{k}^{T} s_{k-1}}{d_{k-1}^{T} y_{k-1}} .
$$


Two further developments of the Dai and Liao's method were made by Yabe and Takano [20] and Li et al. [17] based on different modified secant equations. Some more efficient Dai-Liao-type methods were designed and studied in $[2,9,22,23]$ by using different techniques. In this paper, we further give a new family of DaiLiao-type conjugate gradient methods for unconstrained optimization problems, including their convergence analysis. Numerical experiments show that our methods can outperform the existing ones.

The rest of this paper is organized as follows. In Section 2, we introduce a new Dai-Liao-type method by modifying the conjugate gradient parameter. Based on the strong Wolfe line search rules, the global convergence for uniformly convex and general objective functions is studied in Section 3 and numerical experiments are performed in Section 4. Finally, in Section 5, we give some conclusions to end this paper.

\section{NeW DAi-LiaO-Type Methods}

We start with the original Dai and Liao's method in which the quasi-Newton techniques are used. In the quasi-Newton method, an approximation Hessian $B_{k}$ is updated such that

$$
B_{k} s_{k-1}=y_{k-1}
$$

and the search direction $d_{k}$ is calculated by

$$
d_{k}=-B_{k}^{-1} g_{k}
$$

Combining the above two equations, we have

$$
d_{k}^{T} y_{k-1}=-g_{k}^{T} s_{k-1} .
$$

The above relation implies that (1.7) holds in case of $g_{k}^{T} d_{k-1}=0$, i.e. the line search is exact. However, in practical numerical algorithms, the inexact line search is adopted instead of exact line search. Dai and Liao suggested the following conjugacy condition:

$$
d_{k}^{T} y_{k-1}=-t g_{k}^{T} s_{k-1},(t \geq 0)
$$

In 2004, Yabe and Takano [20] used the modified secant equation

$$
B_{k+1} s_{k}=y_{k}+\rho_{k} \theta_{k} \frac{u_{k}}{u_{k}^{T} s_{k}}
$$

where $\rho_{k} \in[0,3]$ and $\theta_{k}=2\left(f_{k}-f_{k+1}\right)+\left(g_{k}+g_{k+1}\right)^{T} s_{k}, u_{k}$ is chosen s.t. $u_{k}^{T} s_{k} \neq 0$, to derive a new conjugacy condition through replacing $y_{k}$ by $z_{k}=y_{k}+\rho_{k} \theta_{k} \frac{u_{k}}{u_{k}^{T} s_{k}}$, the modified conjugacy parameter is

$$
\beta_{k+1}^{Y T+}=\max \left\{\frac{g_{k+1}^{T} z_{k}}{d_{k}^{T} z_{k}}, 0\right\}-t \frac{g_{k+1}^{T} s_{k}}{d_{k}^{T} z_{k}} .
$$

In this paper, we will derive a new conjugacy condition from another view of point. Combining (2.2) with (2.1), we have

$$
\begin{aligned}
d_{k+1}^{T} y_{k} & =d_{k+1}^{T}\left(B_{k+1} s_{k}-\rho_{k} \theta_{k} \frac{u_{k}}{u_{k}^{T} s_{k}}\right) \\
& =d_{k+1}^{T} B_{k+1} s_{k}-\rho_{k} \theta_{k} \frac{u_{k}^{T} d_{k+1}}{u_{k}^{T} s_{k}} \\
& =-g_{k+1}^{T} s_{k}-\rho_{k} \theta_{k} \frac{u_{k}^{T} d_{k+1}}{u_{k}^{T} s_{k}} .
\end{aligned}
$$


Using the Dai-Liao's conjugacy condition

$$
d_{k+1}^{T} y_{k}=-t g_{k+1}^{T} s_{k}
$$

and $d_{k+1}=-g_{k+1}+\beta_{k+1} d_{k}$, where $t \in[0,1]$, we have

$$
\rho_{k} \theta_{k} \frac{u_{k}^{T}\left(-g_{k+1}+\beta_{k+1} d_{k}\right)}{u_{k}^{T} s_{k}}=(t-1) g_{k+1}^{T} s_{k},
$$

which yields a new conjugate gradient parameter

$$
\begin{aligned}
\beta_{k+1}^{\text {new }} & =\left[\frac{(t-1) g_{k+1}^{T} s_{k} \cdot u_{k}^{T} s_{k}}{\rho_{k} \theta_{k}}+g_{k+1}^{T} u_{k}\right] / d_{k}^{T} u_{k} \\
& =\frac{g_{k+1}^{T} u_{k}}{d_{k}^{T} u_{k}}-(1-t) \frac{u_{k}^{T} s_{k}}{\rho_{k} \theta_{k}} \cdot \frac{g_{k+1}^{T} s_{k}}{d_{k}^{T} u_{k}}
\end{aligned}
$$

if $\rho_{k} \theta_{k} \neq 0$, otherwise, Dai and Liao's conjugate gradient parameter $\beta_{k}^{\mathrm{DL}+}$ will be used. According to the experience of the quasi-Newton methods with modified secant equations [21], we choose $u_{k}=y_{k}$.

In the case of $u_{k}=y_{k}$, the conjugacy parameter $\beta_{k+1}^{\text {new }}$ can be written as

$$
\begin{aligned}
\beta_{k+1}^{\text {new }} & =\left[\frac{(t-1) g_{k+1}^{T} s_{k} \cdot y_{k}^{T} s_{k}}{\rho_{k} \theta_{k}}+g_{k+1}^{T} y_{k}\right] / d_{k}^{T} y_{k} \\
& =\frac{g_{k+1}^{T} y_{k}}{d_{k}^{T} y_{k}}+\frac{(t-1) y_{k}^{T} s_{k}}{\rho_{k} \theta_{k}} \cdot \frac{g_{k+1}^{T} s_{k}}{d_{k}^{T} y_{k}} .
\end{aligned}
$$

and we correct it as

$$
\beta_{k+1}^{\text {new }+}=\max \left\{\frac{g_{k+1}^{T} y_{k}}{d_{k}^{T} y_{k}}, 0\right\}+\frac{(t-1) y_{k}^{T} s_{k}}{\rho_{k}\left|\theta_{k}\right|} \cdot \frac{g_{k+1}^{T} s_{k}}{d_{k}^{T} y_{k}} .
$$

We call the method (1.2) and (1.3) with $\beta_{k}$ given in (2.4) NEW+ method. The corresponding algorithm is given as below:

Algorithm 2.1. Improved Dai-Liao conjugate gradient method

Step 1: Given $x_{0} \in R^{n}, \varepsilon, \eta>0$, set $d_{0}=-g_{0}, k:=0$; if $\left\|g_{0}\right\| \leq \varepsilon$, then stop;

Step 2: Compute $\alpha_{k}$ such that strong Wolfe line search (1.4) and (1.5) hold;

Step 3: Let $x_{k+1}=x_{k}+\alpha_{k} d_{k}$, if $\left\|g_{k+1}\right\| \leq \varepsilon$, then stop;

Step 4: Compute $\beta_{k+1}$ by (2.4) if $\left|\theta_{k}\right|>\eta$, otherwise, compute $\beta_{k+1}$ by (1.9); generate $d_{k+1}$ by (1.3);

Step 5: Set $k:=k+1$ and go to Step 2 .

In the rest of the paper, we first analyze the convergence properties of the new algorithm, then give some numerical results which show the modified algorithms are robust and efficient.

\section{Convergence Analysis}

Throughout this section, we assume that $g_{k} \neq 0$ for all $k \geq 0$, otherwise a stationary point is found. We first give some standard assumptions. 
Assumption 3.1. The level set $\mathcal{L}=\left\{x \in \mathbb{R}^{n}: f(x) \leq f\left(x_{0}\right)\right\}$ is bounded, where $x_{0} \in \mathbb{R}^{n}$ is an initial point.

Assumption 3.2. In some neighborhood $\mathcal{N}$ of $\mathcal{L}$, the function $f$ is continuously differentiable and its gradient $g(x)$ is Lipschitz continuous, i.e. there exists a positive constant $L>0$ such that $\|g(x)-g(y)\| \leq L\|x-y\|$ for all $x, y \in \mathcal{N}$.

Assumption 3.1 guarantees that there exists some constant $c$ such that $\left\|s_{k}\right\| \leq 2 \bar{c}, \forall k>0$. Assumption 3.2 implies that $\|g\| \leq \bar{\gamma}$ for any $x \in \mathcal{L}$, where $\bar{\gamma}=2 \bar{c} L+\left\|g_{0}\right\|$.

Firstly, we give some estimation on $\theta_{k}$. We know by mean value theorem that

$$
\begin{aligned}
\theta_{k} & =2\left(f_{k}-f_{k+1}\right)+\left(g_{k}+g_{k+1}\right)^{T} s_{k} \\
& =-2 \nabla f\left(\eta_{k}\right)^{T} s_{k}+\left(g_{k}+g_{k+1}\right)^{T} s_{k} \\
& =-\left[\nabla f\left(x_{k}\right)-\nabla f\left(\eta_{k}\right)+\nabla f\left(x_{k+1}\right)-\nabla f\left(\eta_{k}\right)\right]^{T} s_{k},
\end{aligned}
$$

where $\eta_{k}=x_{k}+\tau\left(x_{k+1}-x_{k}\right)$ and $\tau \in(0,1)$. Hence

$$
\begin{aligned}
\left|\theta_{k}\right| & \leq\left(\left\|\nabla f\left(x_{k}\right)-\nabla f\left(\eta_{k}\right)\right\|+\left\|\nabla f\left(x_{k+1}\right)-\nabla f\left(\eta_{k}\right)\right\|\right)\left\|s_{k}\right\| \\
& \leq L\left(\left\|x_{k}-\eta_{k}\right\|+\left\|x_{k+1}-\eta_{k}\right\|\right)\left\|s_{k}\right\| \\
& =L\left\|s_{k}\right\|^{2} \leq 4 L \bar{c}^{2} .
\end{aligned}
$$

On the other hand, since $\theta_{k}$ is appeared in the denominator, too small value must be avoided for the numerical stability, we ask $\left|\theta_{k}\right|$ to satisfy $0<\eta \leq\left|\theta_{k}\right|$ as shown in Algorithm 2.1. Otherwise, $\beta_{k}^{\text {DL+ }}$ will be used.

Let $f$ be a uniformly convex function, then there exists some constant $\mu>0$ such that

$$
(\nabla f(x)-\nabla f(y))^{T}(x-y) \geq \mu\|y-x\|^{2}
$$

which implies

$$
\mu\left\|s_{k}\right\|^{2} \leq s_{k}^{T} y_{k} \leq L\left\|s_{k}\right\|^{2}
$$

Then we have that

$$
\begin{aligned}
\theta_{k} & =2\left(f_{k}-f_{k+1}\right)+\left(g_{k}+g_{k+1}\right)^{T} s_{k} \\
& \geq\left(-g_{k+1}^{T} s_{k}+\frac{\mu}{2}\left\|s_{k}\right\|^{2}\right)+\left(g_{k}+g_{k+1}\right)^{T} s_{k} \\
& =-s_{k}^{T} y_{k}+\mu\left\|s_{k}\right\|^{2} \\
& \geq-\left(1-\frac{\mu}{L}\right) s_{k}^{T} y_{k}
\end{aligned}
$$

and

$$
\left|\theta_{k}\right| \leq L\left\|s_{k}\right\|^{2} \leq \frac{L}{\mu} s_{k}^{T} y_{k}
$$

Thus $\theta_{k}$ locates in the interval $\left(-(1-\mu / L) s_{k}^{T} y_{k},-\eta\right) \cup\left(\eta, L / \mu s_{k}^{T} y_{k}\right)$. Therefore, we assume that the following relationship always holds.

$$
\frac{\mu}{L} \leq\left|\frac{s_{k}^{T} y_{k}}{\theta_{k}}\right| \leq \frac{1}{\epsilon}
$$

The following theorem states the global convergence property of new method for uniformly convex functions.

Theorem 3.3. Let $f$ be a uniformly convex function and Assumptions 3.1 and 3.2 hold. Suppose that $\left\{x_{k}\right\}$ is the sequence generated by Algorithm 2.1 with $\beta_{k}$ in (2.4), then

$$
\lim _{k \rightarrow \infty}\left\|g_{k}\right\|=0 .
$$


Proof. It follows from $f$ is uniformly convex function that

$$
d_{k}^{T} y_{k} \geq \mu \alpha_{k-1}\left\|d_{k-1}\right\|^{2} .
$$

By using Triangular and Cauchy-Schwartz inequalities, we have

$$
\begin{aligned}
\left\|d_{k}\right\| & \leq\left\|g_{k}\right\|+\left|\beta_{k}^{\text {new }}\right|\left\|d_{k-1}\right\| \\
& \leq\left\|g_{k}\right\|+\frac{(L+(1-t) / \epsilon)\left\|g_{k}\right\|\left\|s_{k-1}\right\|}{\mu \alpha_{k-1}\left\|d_{k-1}\right\|^{2}}\left\|d_{k-1}\right\| \\
& \leq \mu^{-1}(L+(1-t) / \epsilon+\mu)\left\|g_{k}\right\|,
\end{aligned}
$$

which means

$$
\sum_{k \geq 1} \frac{1}{\left\|d_{k}\right\|^{2}}=+\infty
$$

Therefore, from Lemma $3.2[6]$ and the fact $f$ is a uniformly convex function, we have

$$
\lim _{k \rightarrow \infty}\left\|g_{k}\right\|=0 \text {. }
$$

For the general function, we only need to show the modified Dai-Liao method with $\beta_{k}$ in (2.4) satisfies the Property $(*)$ depicted by Gilbert and Nocedal [10]. The rest analysis is similar to the original Dai-Liao's method.

Definition 3.4. Consider a method of the form (1.2)-(1.3), and suppose that

$$
0<\gamma \leq\left\|g_{k}\right\| \leq \bar{\gamma}
$$

for all $k \geq 1$. We say that the conjugate gradient method has the Property $(*)$, if for all $k$, there exist constants $b>1, k>0$ such that for all $k$,

$$
\left|\beta_{k}\right| \leq b \text { and }\left\|s_{k-1}\right\| \leq \lambda \text { imply }\left|\beta_{k}\right| \leq \frac{1}{2 b} .
$$

By the strong Wolfe condition (1.5), (1.10) and (3.3), we have

$$
d_{k-1}^{T} y_{k-1} \geq(\sigma-1) g_{k-1}^{T} d_{k-1} \geq(1-\sigma) c \gamma^{2} .
$$

Using this and boundedness of $\left\|s_{k}\right\|$, we obtain

$$
\left|\beta_{k}\right| \leq \frac{(L+(1-t) / \epsilon)\left\|g_{k}\right\|\left\|s_{k-1}\right\|}{(1-\sigma) c \gamma^{2}} \leq \frac{2(L+(1-t) / \epsilon) \bar{\gamma} \bar{c}}{(1-\sigma) c \gamma^{2}}=: b .
$$

Note that $b$ can be defined such that $b>1$. If we set

$$
\lambda:=\frac{(1-\sigma) c \gamma^{2}}{b(L+(1-t) / \epsilon) \bar{\gamma}}
$$

and $s_{k-1} \leq \lambda$, then

$$
\left|\beta_{k}\right| \leq \frac{(L+(1-t) / \epsilon) \bar{\gamma} \lambda}{(1-\sigma) c \gamma^{2}}=\frac{1}{b}
$$

Therefore, the NEW+ method has Property $\left(^{*}\right)$. Thus, we have the following convergence theorem.

Theorem 3.5. Let Assumptions 3.1 and 3.2 hold. If the sequence $\left\{x_{k}\right\}$ is generated by the $N E W+$ method with the strong Wolfe line search for $\sigma \in(0,1)$, where $d_{k}$ satisfies condition $(1.10)$ with $c>0$. Then we have

$$
\liminf _{k \rightarrow+\infty}\left\|g_{k}\right\|=0
$$


TABLE 1. Tested conjugate gradient algorithms.

\begin{tabular}{lll}
\hline \hline$\beta_{k}$ & Name of method & Abbreviation \\
\hline$\beta_{k}^{\mathrm{FR}}$ & The Fletcher-Reeves method [8] & FR \\
$\beta_{k}^{\mathrm{PRP}+}$ & The Polak-Ribiere-Polyak [18, 19] & PRP + \\
$\beta_{k}^{\mathrm{HS}}$ & The Hestenes-Stiefel method [14] & HS \\
$\beta_{k}^{\mathrm{DY}}$ & The Dai-Yuan method [3] & $\mathrm{DY}$ \\
$\beta_{k}^{\mathrm{N}}$ & The Hager and Zhang's method [12] & HZ \\
$\beta_{k}^{\mathrm{DL}+}$ & The Dai-Liao method [6] & DL+ \\
$\beta_{k}^{\mathrm{YT}+}$ & Yabe-Takano's method [20] & YT + \\
$\beta_{k}^{\text {new }+}$ & Our method & NEW + \\
\hline
\end{tabular}

TABLE 2. Numerical results for Trigonometric with $n=5000$.

\begin{tabular}{|c|c|c|c|c|c|c|}
\hline FR & \multicolumn{2}{|c|}{$\mathrm{PRP}+$} & HS & DY & $\mathrm{HZ}$ & CG_descent \\
\hline \multirow[t]{3}{*}{$167 / 292$} & \multicolumn{2}{|c|}{$133 / 340$} & $39 / 109$ & $213 / 291$ & $96 / 195$ & $73 / 278$ \\
\hline & \multirow{2}{*}{$\rho_{k}$} & \multicolumn{5}{|c|}{$t$} \\
\hline & & 0.1 & 0.3 & 0.5 & 0.7 & 0.9 \\
\hline DL+ & 0 & $21 / 100$ & $49 / 136$ & $20 / 92$ & $64 / 156$ & $69 / 158$ \\
\hline YT+ & \multirow{2}{*}{0.1} & $21 / 100$ & $82 / 177$ & $20 / 92$ & $61 / 152$ & $63 / 151$ \\
\hline NEW+ & & $78 / 169$ & $61 / 143$ & $3 \quad 52 / 136$ & $19 / 91$ & $66 / 156$ \\
\hline YT+ & \multirow{2}{*}{0.3} & $20 / 99$ & $67 / 160$ & $20 / 93$ & $21 / 95$ & $63 / 153$ \\
\hline NEW & & $20 / 94$ & $20 / 93$ & $20 / 91$ & $65 / 157$ & $67 / 161$ \\
\hline YT+ & \multirow{2}{*}{0.5} & $19 / 95$ & $21 / 91$ & $42 / 134$ & $41 / 127$ & $38 / 127$ \\
\hline NEW+ & & $20 / 92$ & $19 / 88$ & $19 / 89$ & $64 / 155$ & $71 / 162$ \\
\hline YT+ & \multirow{2}{*}{0.7} & $22 / 107$ & $21 / 93$ & $67 / 154$ & $69 / 162$ & $23 / 100$ \\
\hline NEW+ & & $22 / 108$ & $45 / 135$ & $20 / 91$ & $64 / 152$ & $64 / 152$ \\
\hline $\mathrm{YT}+$ & \multirow{2}{*}{0.9} & $20 / 95$ & $68 / 166$ & $21 / 99$ & $38 / 127$ & $33 / 1$ \\
\hline NEW+ & & $20 / 97$ & $47 / 140$ & $20 / 98$ & $67 / 158$ & $19 / 94$ \\
\hline YT+ & \multirow{2}{*}{1.0} & $21 / 97$ & $20 / 90$ & $21 / 95$ & $40 / 132$ & $22 / 95$ \\
\hline NEW+ & & $20 / 97$ & $44 / 128$ & $19 / 89$ & $65 / 153$ & $19 / 95$ \\
\hline
\end{tabular}

\section{NumERICAL EXPERIMENTS}

In this section, some numerical results are reported on a a set of 76 unconstrained optimization problems selected from [1] and CUTEst library [11]. We tested the conjugate gradient algorithms with the conjugacy parameters given in Table 1.

For the algorithms DL+, YT + and our new method, different scaled parameters $\rho$ and $t$ are used. In the case where an ascent direction is generated, we restart the algorithm by setting $d_{k}=-g_{k}$.

All codes were written in Fortran and in double precision arithmetic. (Note that, for the sake of fairness, at the beginning of experiments we do not directly run Hager and Zhang's CG_descent codes for the test problems, we just use their conjugate parameter under the same linear search in our test framework). The stopping rule is set as $\left\|g_{k}\right\|_{\infty} \leq 10^{-6}$. The iteration is also terminated if the total number of iterations exceeds 10,000 . Partial numerical results are summarized in Tables 2-6 and given in the form of (number of iterations/number of function-gradient evaluations), the detailed complete numerical results can be downloaded from the website https://github.com/piratetwo/mdl. 
TABLE 3. Numerical results for ENGVAL1 with $n=5000$.

\begin{tabular}{|c|c|c|c|c|c|c|}
\hline FR & \multicolumn{2}{|c|}{$\mathrm{PRP}+$} & $\mathrm{HS}$ & DY & $\mathrm{HZ}$ & CG_descent \\
\hline \multirow{3}{*}{$285 / 8484$} & \multicolumn{2}{|c|}{$195 / 5333$} & $695 / 20246$ & $1711 / 53963$ & $229 / 6770$ & $24 / 78$ \\
\hline & \multirow{2}{*}{$\rho_{k}$} & \multicolumn{5}{|c|}{$t$} \\
\hline & & 0.1 & 0.3 & 0.5 & 0.7 & 0.9 \\
\hline DL+ & 0 & $117 / 3068$ & $33 / 364$ & $24 / 72$ & $105 / 2642$ & $161 / 4520$ \\
\hline YT+ & $C_{1}$ & $101 / 2570$ & $224 / 6557$ & $145 / 3867$ & $105 / 2774$ & $43 / 704$ \\
\hline NEW+ & 0.1 & $189 / 5398$ & $162 / 4586$ & $93 / 2162$ & $197 / 5752$ & $269 / 7994$ \\
\hline$\overline{\mathrm{YT}+}$ & & $166 / 4696$ & $185 / 5189$ & $184 / 5306$ & $112 / 2886$ & $181 / 5142$ \\
\hline NEW+ & 0.3 & $228 / 6821$ & $200 / 6005$ & $307 / 9366$ & $137 / 3683$ & $255 / 7464$ \\
\hline $\mathrm{YT}+$ & & $179 / 5123$ & $171 / 4828$ & $157 / 4448$ & $140 / 3857$ & $136 / 3666$ \\
\hline NEW+ & 0. & $162 / 4693$ & $164 / 4715$ & $170 / 4931$ & $78 / 1778$ & $144 / 3926$ \\
\hline YT+ & & $58 / 1225$ & $79 / 1805$ & $81 / 1896$ & $217 / 6311$ & $164 / 4696$ \\
\hline NEW+ & 0.7 & $46 / 805$ & $253 / 7499$ & $126 / 3339$ & $157 / 4211$ & $128 / 3529$ \\
\hline $\mathrm{YT}+$ & & $200 / 6064$ & $127 / 3524$ & $60 / 1233$ & $149 / 4241$ & $259 / 7689$ \\
\hline NEW+ & & $37 / 431$ & $177 / 4856$ & $72 / 1552$ & $234 / 6807$ & $116 / 2866$ \\
\hline $\mathrm{YT}+$ & & $120 / 3187$ & $159 / 4454$ & $109 / 2979$ & $282 / 8316$ & $145 / 4029$ \\
\hline NEW+ & 1.0 & $94 / 2333$ & $62 / 1125$ & $120 / 3091$ & $102 / 2489$ & $119 / 3154$ \\
\hline
\end{tabular}

TABLE 4. Numerical results for Raydan 1 with $n=5000$.

\begin{tabular}{|c|c|c|c|c|c|c|}
\hline FR & $\mathrm{PRP}+$ & HS & DY & $\mathrm{HZ}$ & CG_descent & \\
\hline \multirow[t]{3}{*}{$-1-$} & $-1-$ & $756 / 1019$ & $739 / 819$ & $856 / 1334$ & $490 / 1472$ & \\
\hline & \multirow[b]{2}{*}{$\rho_{k}$} & \multicolumn{5}{|c|}{$t$} \\
\hline & & 0.1 & 0.3 & 0.5 & 0.7 & 0.9 \\
\hline DL+ & 0 & $816 / 1077$ & $782 / 1082$ & $724 / 972$ & $766 / 1046$ & $812 / 1101$ \\
\hline $\mathrm{YT}+$ & \multirow{2}{*}{0.1} & $834 / 1274$ & $770 / 1041$ & $766 / 1077$ & $540 / 711$ & $767 / 1001$ \\
\hline NEW+ & & $850 / 1166$ & $689 / 933$ & $808 / 1092$ & $649 / 853$ & $785 / 1037$ \\
\hline $\mathrm{YT}+$ & \multirow{2}{*}{0.3} & $809 / 1109$ & $749 / 1038$ & $782 / 1063$ & $810 / 1122$ & $863 / 1140$ \\
\hline NEW+ & & $839 / 1142$ & $628 / 858$ & $748 / 1010$ & $791 / 1063$ & $864 / 1154$ \\
\hline YT+ & \multirow{2}{*}{0.5} & $752 / 1032$ & $744 / 1015$ & $749 / 1028$ & $724 / 964$ & $829 / 1135$ \\
\hline NEW+ & & $764 / 1015$ & $732 / 1011$ & $779 / 1058$ & $710 / 964$ & $801 / 1090$ \\
\hline $\mathrm{YT}+$ & \multirow{2}{*}{0.7} & $622 / 853$ & $679 / 915$ & $826 / 1144$ & $842 / 1114$ & $789 / 1065$ \\
\hline NEW+ & & $719 / 970$ & $736 / 992$ & $704 / 953$ & $696 / 940$ & $820 / 1081$ \\
\hline YT+ & \multirow[b]{2}{*}{0.9} & $712 / 974$ & $824 / 1094$ & $882 / 1224$ & $777 / 1045$ & $890 / 1187$ \\
\hline NEW+ & & $671 / 891$ & $870 / 1151$ & $786 / 1062$ & $851 / 1179$ & $746 / 991$ \\
\hline YT+ & \multirow{2}{*}{1.0} & $708 / 957$ & $859 / 1183$ & $669 / 897$ & $876 / 1176$ & $815 / 1103$ \\
\hline NEW+ & & $722 / 969$ & $715 / 972$ & $759 / 1023$ & $706 / 948$ & $680 / 905$ \\
\hline
\end{tabular}

In the Tables 2-6, the boldface font is used to mark the first and second efficient method which performs better than the other two algorithms for each $\rho_{k}$ and $t$. The number of the best performance for Algorithm $\mathrm{NEW}+, \mathrm{YT}+, \mathrm{DL}+$ are 32, 15 and 3, respectively.

In most cases, our new method improves Yabe-Takano's method. For a special $\rho_{k}=3$, which was used in the modified quasi-Newton method, we compare the numerical performance of YT+ and NEW+. We run the codes with different $t=0.1,0.2, \ldots, 1$ and compute the medians for each problem. The performance profiles introduced by Dolan and Moré [7] are used to display the behaviours of these two methods. Figure 1 shows that the NEW+ method performs the best result regarding the number of iterations and function-gradient evaluations, which is located at the top curve in Figure 1. 
TABLE 5. Numerical results for SINQUAD with $n=5000$.

\begin{tabular}{|c|c|c|c|c|c|c|c|}
\hline $\mathrm{FR}$ & & $\mathrm{RP}+$ & $\mathrm{HS}$ & DY & $\mathrm{HZ}$ & CG_descent & \\
\hline \multirow[t]{3}{*}{$1455 / 2665$} & & $-1-$ & $-/-$ & $1891 / 2579$ & $2021 / 4044$ & $1071 / 3813$ & \\
\hline & \multirow[b]{2}{*}{$\rho_{k}$} & \multicolumn{6}{|c|}{$t$} \\
\hline & & & 0.1 & 0.3 & 0.5 & 0.7 & 0.9 \\
\hline DL+ & 0 & & $481 / 1014$ & $681 / 1424$ & $728 / 1532$ & $475 / 1018$ & $588 / 1238$ \\
\hline \multirow{2}{*}{$\begin{array}{l}\mathrm{YT}+ \\
\mathrm{NEW}+\end{array}$} & \multirow{2}{*}{0.1} & 602 & $/ 1270$ & $499 / 1043$ & $792 / 1676$ & $703 / 1515$ & $390 / 874$ \\
\hline & & 550 & $/ 1172$ & $530 / 1145$ & $465 / 1021$ & $451 / 965$ & $606 / 1282$ \\
\hline \multirow{2}{*}{$\begin{array}{l}\mathrm{YT}+ \\
\mathrm{NEW}+\end{array}$} & \multirow{2}{*}{0.3} & \multicolumn{2}{|c|}{$533 / 1121$} & $568 / 1225$ & $1431 / 2951$ & $477 / 1013$ & $526 / 1110$ \\
\hline & & 482 & /1009 & $721 / 1507$ & $638 / 1325$ & $599 / 1277$ & $597 / 1249$ \\
\hline \multirow{2}{*}{$\begin{array}{l}\mathrm{YT}+ \\
\mathrm{NEW}+\end{array}$} & \multirow{2}{*}{0.5} & \multicolumn{2}{|c|}{$528 / 1095$} & $579 / 1272$ & $544 / 1210$ & $687 / 1474$ & $553 / 1185$ \\
\hline & & 532 & /1132 & $491 / 1057$ & $804 / 1682$ & $503 / 1059$ & $500 / 1094$ \\
\hline \multirow{2}{*}{$\begin{array}{l}\mathrm{YT}+ \\
\mathrm{NEW}+\end{array}$} & \multirow{2}{*}{0.7} & \multicolumn{2}{|c|}{$588 / 1295$} & $842 / 1773$ & $496 / 1048$ & $555 / 1197$ & $499 / 1102$ \\
\hline & & & 1105 & $543 / 1195$ & $565 / 1189$ & $452 / 1158$ & $476 / 1012$ \\
\hline \multirow{2}{*}{$\begin{array}{l}\mathrm{YT}+ \\
\text { NEW+ }\end{array}$} & \multirow{2}{*}{0.9} & \multicolumn{2}{|c|}{$\begin{array}{l}553 / 1105 \\
546 / 1212\end{array}$} & $522 / 1088$ & $610 / 1275$ & $598 / 1287$ & $568 / 1235$ \\
\hline & & 483 & $/ 1029$ & $594 / 1280$ & $502 / 1068$ & $489 / 1046$ & $644 / 1358$ \\
\hline YT+ & \multirow{2}{*}{1.0} & 423 & $3 / 873$ & $590 / 1272$ & $538 / 1162$ & $498 / 1048$ & $483 / 1011$ \\
\hline NEW+ & & 593 & /1275 & $526 / 1119$ & $567 / 1182$ & $569 / 1204$ & $506 / 1064$ \\
\hline
\end{tabular}

TABLE 6. Numerical results for Woods with $n=5000$.

\begin{tabular}{lcccccc}
\hline \hline FR & PRP+ & HS & DY & HZ & CG_descent \\
\hline \multirow{2}{*}{$112 / 218$} & \multirow{2}{*}{$65 / 133$} & $87 / 214$ & $95 / 178$ & $161 / 309$ & $182 / 683$ \\
\hline & \multirow{2}{*}{$\rho_{k}$} & 0.1 & 0.3 & 0.5 & 0.7 & 0.9 \\
\cline { 3 - 8 } DL+ & 0 & $39 / 80$ & $52 / 99$ & $43 / 85$ & $45 / 90$ & $48 / 94$ \\
\hline YT+ & \multirow{2}{*}{0.1} & $\mathbf{3 2 / 6 8}$ & $37 / 76$ & $63 / 122$ & $59 / 113$ & $46 / 90$ \\
NEW+ & & $38 / 77$ & $\mathbf{3 7 / 7 5}$ & $70 / 123$ & $43 / 90$ & $44 / 89$ \\
\hline YT+ & \multirow{2}{*}{0.3} & $48 / 93$ & $40 / 101$ & $53 / 103$ & $58 / 116$ & $61 / 114$ \\
NEW+ & \multirow{2}{*}{$39 / 83$} & $44 / 86$ & $48 / 97$ & $46 / 96$ & $\mathbf{3 6 / 7 4}$ \\
\hline YT+ & \multirow{2}{*}{0.5} & $32 / 69$ & $42 / 83$ & $41 / 80$ & $81 / 161$ & $58 / 116$ \\
NEW+ & & $62 / 113$ & $\mathbf{3 4 / 7 2}$ & $50 / 100$ & $\mathbf{3 6 / 7 8}$ & $53 / 103$ \\
\hline YT+ & \multirow{2}{*}{0.7} & $35 / 74$ & $49 / 99$ & $40 / 81$ & $58 / 114$ & $45 / 91$ \\
NEW+ & & $35 / 76$ & $51 / 103$ & $\mathbf{3 6 / 7 0}$ & $69 / 132$ & $58 / 109$ \\
\hline YT+ & \multirow{2}{*}{0.9} & $\mathbf{3 1 / 6 6}$ & $44 / 86$ & $47 / 93$ & $38 / 79$ & $\mathbf{3 9 / 7 9}$ \\
NEW+ & & $40 / 83$ & $49 / 97$ & $57 / 108$ & $\mathbf{3 6 / 7 4}$ & $59 / 99$ \\
\hline YT+ & \multirow{2}{*}{1.0} & $41 / 83$ & $41 / 83$ & $36 / 74$ & $39 / 79$ & $59 / 118$ \\
NEW+ & & $48 / 95$ & $60 / 113$ & $\mathbf{3 2 / 6 7}$ & $46 / 91$ & $41 / 84$ \\
\hline
\end{tabular}

Finally, we run Hager and Zhang's method with the approximate Wolfe line search conditions (Hager and Zhang's CG_descent Fortran code Version $1.4^{2}$ ). From Figure 2, for about $62 \%$ of all problems, CG_descent needs the least iterations, it has the best performance. However, CG_descent has the poorer performance than the $\mathrm{YT}+$ and NEW+ regarding the number of function-gradient evaluations which mainly affects the efficiency of the methods.

\footnotetext{
$2_{\text {https : //people.clas.ufl.edu/hager/software/ }}$
} 

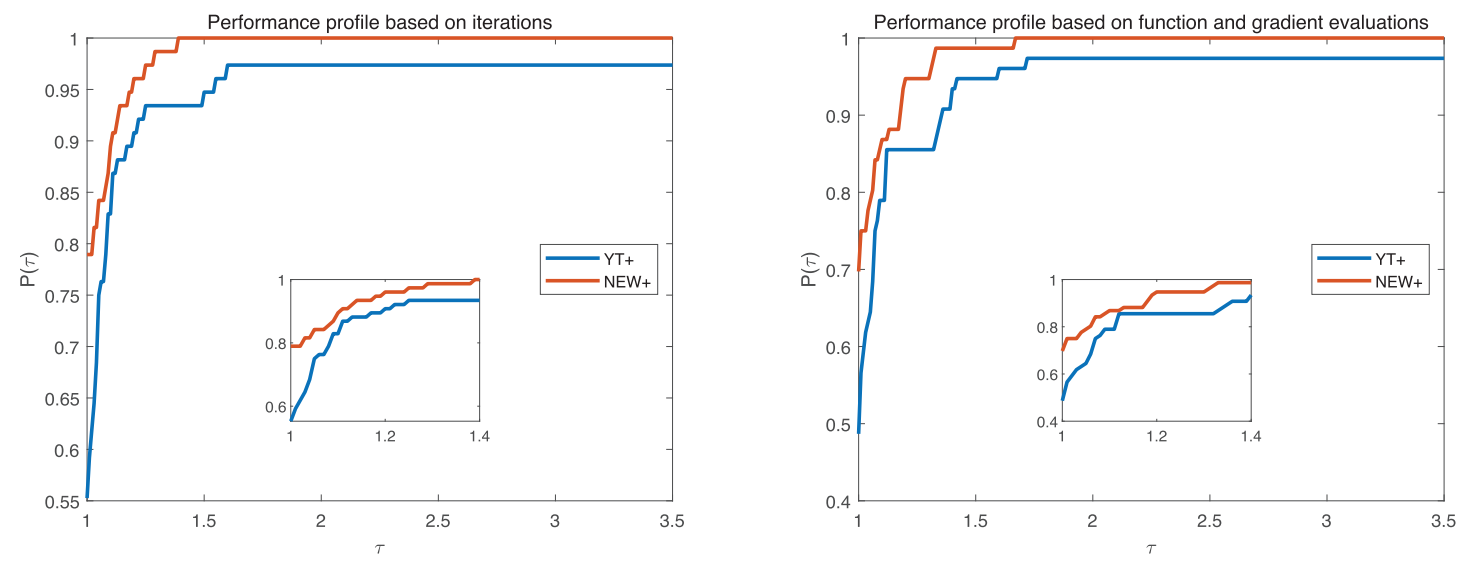

FIgURE 1. Performance profiles based on iterations and function-gradient evaluations for YT+ and NEW+.
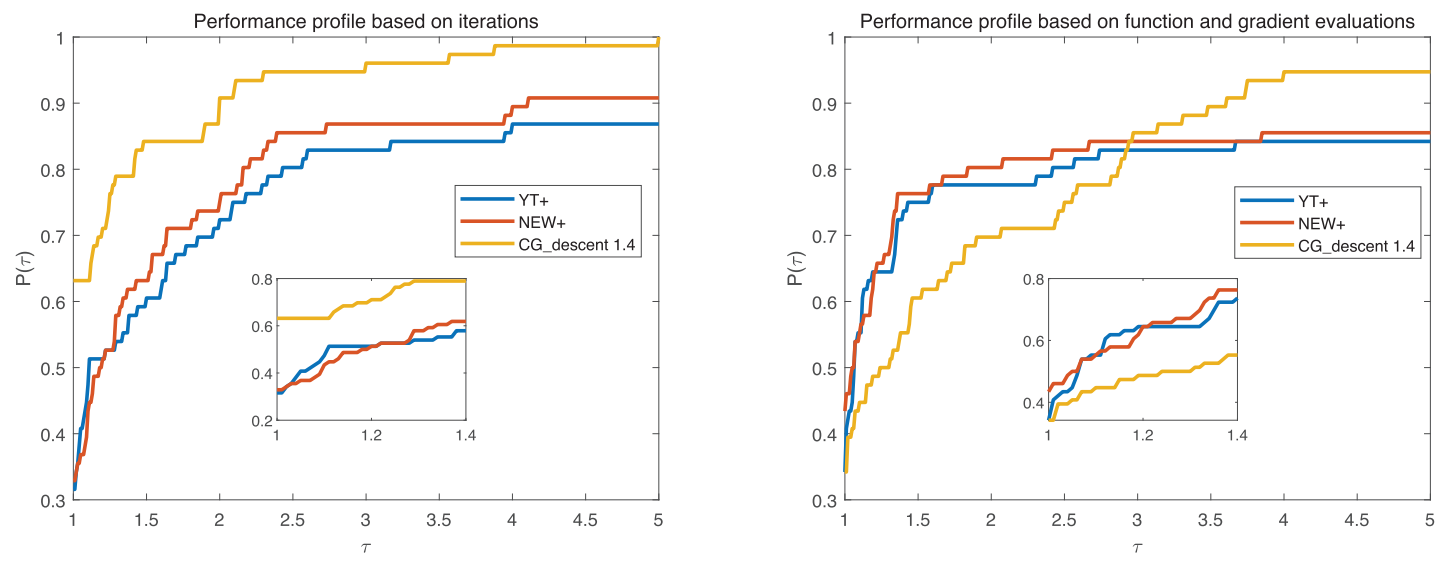

FIgURE 2. Performance profiles based on iterations and function-gradient evaluations for YT+, NEW+ and CG_descent.

\section{Conclusions}

In this paper, based on the Dai and Liao's conjugacy condition and the modified secant condition proposed by Zhang and $\mathrm{Xu}$ [21], we derived a new family of Dai-Liao-type conjugate gradient methods. Under some certain assumptions, we show that our methods are globally convergent for general functions. Numerical results show that our new methods can outperform some existing ones.

Acknowledgements. This work was supported by the Natural Science Foundation of Henan Province (Grant No. 202300410236) and the Training Program Foundation for Youth of Henan Normal University (Grant No. 2020PL28).

\section{REFERENCES}

[1] N. Andrei, An unconstrained optimization test functions collection. Adv. Model. Optim. 10 (2008) 147-161.

[2] Y. Cheng, Q. Mou, X. Pan and S. Yao, A sufficient descent conjugate gradient method and its global convergence. Optim. Methods Softw. 31 (2016) 577-590. 
[3] Y. Dai and Y. Yuan, A nonlinear conjugate gradient method with a strong global convergence property. SIAM J. Optim. 10 (1999) $177-182$.

[4] Y. Dai, Nonlinear conjugate gradient methods, in Wiley Encyclopedia of Operations Research and Management Science (2011). DOI: $10.1002 / 9780470400531$.eorms 0183

[5] Y. Dai, Y. Huang and X. Liu, A family of spectral gradient methods for optimization. Comput. Optim. Appl. 74 (2019) 43-65.

[6] Y. Dai and L. Liao, New conjugacy conditions and related nonlinear conjugate gradient methods. Appl. Math. Optim. 43 (2001) $87-101$.

[7] E. Dolan and J. Moré, Benchmarking optimization software with performance profiles. Math. Program. 91 (2002) $201-213$.

[8] R. Fletcher, Function minimization by conjugate gradients. Comput. J. 7 (1964) 149-154.

[9] J. Ford, Y. Narushima and H. Yabe, Multi-step nonlinear conjugate gradient methods for unconstrained minimization. Comput. Optim. Appl. 40 (2008) 191-216.

[10] J. Gilbert and J. Nocedal, Global convergence properties of conjugate gradient methods for optimization. SIAM J. Optim. 2 (1992) 21-42.

[11] N. Gould, D. Orban and Ph. Toint, CUTEst: a Constrained and Unconstrained Testing Environment with safe threads for mathematical optimization. Comput. Optim. Appl. 60 (2015) 545-557.

[12] W. Hager and H. Zhang, A new conjugate gradient method with guaranteed descent and an efficient line search. SIAM J. Optim. 16 (2005) 170-192.

[13] W. Hager and H. Zhang. A survey of nonlinear conjugate gradient methods. Pacific J. Optim. 2 (2006) 35-58.

[14] M. Hestenes and E. Stiefel, Methods of conjugate gradients for solving linear systems. J. Res. Nat. Bur. Stand. 49 (1952) 409-436.

[15] Y. Huang, Y. Dai, X. Liu and H. Zhang, Gradient methods exploiting spectral properties. Optim. Methods Softw. 35 (2020) 681-705.

[16] C. Kou and Y. Dai, A modified self-scaling memoryless Broyden-Fletcher-Goldfarb-Shanno method for unconstrained optimization. J. Optim. Theory Appl. 165 (2015) 209-224.

[17] G. Li, C. Tang and Z. Wei, New conjugacy condition and related new conjugate gradient methods for unconstrained optimization. J. Comput. Appl. Math. 202 (2007) 523-539.

[18] E. Polak and G. Ribiere, Note sur la convergence de méthodes de directions conjuguées. ESAIM: M2AN 3 (1969) 35-43.

[19] B. Polyak, The conjugate gradient method in extremal problems. USSR Comput. Math. Math. Phys. 9 (1969) 94-112.

[20] H. Yabe and M. Takano, Global convergence properties of nonlinear conjugate gradient methods with modified secant condition. Comput. Optim. Appl. 28 (2004) 203-225.

[21] J. Zhang and C. Xu, Properties and numerical performance of quasi-Newton methods with modified quasi-Newton equations. J. Comput. Appl. Math. 137 (2001) 269-278.

[22] K. Zhang, H. Liu and Z. Liu, A new Dai-Liao conjugate gradient method with optimal parameter choice. Numer. Funct. Anal. Optim. 40 (2019) 194-215.

[23] Y. Zheng and B. Zheng, Two new Dai-Liao-type conjugate gradient methods for unconstrained optimization problems. J. Optim. Theory Appl. 175 (2017) 502-509.

\section{Subscribe to Open (S20) A fair and sustainable open access model}

This journal is currently published in open access under a Subscribe-to-Open model (S2O). S2O is a transformative model that aims to move subscription journals to open access. Open access is the free, immediate, online availability of research articles combined with the rights to use these articles fully in the digital environment. We are thankful to our subscribers and sponsors for making it possible to publish this journal in open access, free of charge for authors.

\section{Please help to maintain this journal in open access!}

Check that your library subscribes to the journal, or make a personal donation to the S2O programme, by contacting subscribers@edpsciences.org

More information, including a list of sponsors and a financial transparency report, available at: https://www. edpsciences.org/en/maths-s2o-programme 